\title{
Key elements of infectious disease syndromic surveillance systems: A scoping review
}

\author{
Stephanie L. Hughes ${ }^{\star 1}$, Alex J. Elliot ${ }^{2}$, Scott A. McEwen ${ }^{1}$, Amy Greer ${ }^{1}$, lan Young ${ }^{3}$ and \\ Andrew Papadopoulos ${ }^{1}$
}

${ }^{1}$ Population Medicine, University of Guelph, Guelph, ON, Canada; ${ }^{2}$ Public Health England, Birmingham, United Kingdom; ${ }^{3}$ Ryerson University, Toronto, ON, Canada

\begin{abstract}
Introduction
Syndromic surveillance is an alternative type of public health surveillance which utilises pre-diagnostic data sources to detect outbreaks earlier than conventional (laboratory) surveillance and monitor the progression of illnesses in populations. These systems are often noted for their ability to detect a wider range of cases in underreported illnesses, utilise existing data sources, and alert public health authorities of emerging crises. In addition, they are highly versatile and can be applied to a wide range of illnesses (communicable and non-communicable) and environmental conditions. As a result, their implementation in public health practice is expanding rapidly. This scoping review aimed to identify all existing literature detailing the necessary components in the defining, creating, implementing, and evaluating stages of human infectious disease syndromic surveillance systems.
\end{abstract}

\section{Methods}

A full scoping review protocol was developed a priori. The research question posed for the review was "What are the essential elements of a fully functional syndromic surveillance system for human infectious disease?" Five bibliographic databases (Pubmed, Scopus, CINAHL, Web of Science, ProQuest) and eleven websites (Google, Public Health Ontario, Public Health England, Public Health Agency of Canada, Centers for Disease Control and Prevention, European Centre for Disease Prevention and Control, International Society for Disease Surveillance, Syndromic Surveillance Systems in Europe, Eurosurveillance, Kingston Frontenac, Lennox \& Addington Public Health (x2)) were searched for peer-reviewed, government, academic, conference, and book literature. A total of 1237 unique citations were identified from this search and uploaded into the scoping review software Covidence. The titles and abstracts were screened for relevance to the subject material, resulting in 142 documents for full-text screening. Following this step, 55 documents remained for data extraction and inclusion in the scoping review. Two independent reviewers conducted each step.

\section{Results}

The scoping review identified many essential elements in the defining, creating, implementing, and evaluating of syndromic surveillance systems. These included the defining of "syndromic surveillance", classification of syndromes, data quality and completeness, statistical methods, privacy and confidentiality issues, costs, operational challenges, management composition, collaboration with other public health agencies, and evaluation criteria. Several benefits and limitations of the systems were also identified, when comparing them to other public health surveillance methods. Benefits included the timeliness of analyses and reporting, potential cost savings, complementing traditional surveillance methods, high sensitivity, versatility, ability to perform short- and long-term surveillance, non-specificity of the systems, ability to fill in gaps of under-reported illnesses, and the collaborations which are fostered through its platform; limitations included the potential resources and costs required, inability to replace traditional healthcare and surveillance methods, the false alerts which may occur, nonspecificity of the systems, poor data quality and completeness, time lags in analyses, limited effectiveness at detecting smaller-scale outbreaks, and privacy issues with accessing data.

\section{Conclusions}

Over the past decade, syndromic surveillance systems have become an integral part of public health practice internationally. Their ability to monitor a wide variety of illnesses and conditions, detect illnesses earlier than traditional surveillance methods, and be created using existing data sources make them a valuable public health tool The results from this scoping review demonstrate the benefits and limitations and overall role of the systems in public health practice. In addition, this study also shows that a complete set of key elements are required in order to properly define, create, implement, and evaluate these systems to ensure their effectiveness and performance.

\section{Keywords}

Syndromic surveillance; Elements; Scoping review

\section{Acknowledgments}

I would like to acknowledge the kindness from Public Health England, Public Health Ontario, Kingston, Frontenac and Lennox \& Addington Public Health, and Sykes Assistance Services Corporation in the development and execution of my project.

\section{*Stephanie L. Hughes}

E-mail: shughes@uoguelph.ca 